\title{
Alimentation des lapins (Oryctolagus cuniculus L.) à base de Brachiaria ruzienzis et Aeschynomene histrix, Stylosanthès hamata et Arachis pintoï sur les performances zootechniques.
}

\author{
SANA Youssoufou1ㅜ, KONDOMBO Salam Richard1 SANOU Jacob1', SAWADOGO Louis ${ }^{13}$, KABORE- \\ ZOUNGRANA Chantal23 \\ ${ }^{1}$ Institut de l'Environnement et de Recherches Agricoles (INERA) 04 BP 8645 Ouagadougou 04 Burkina Faso \\ 2 Université Nazi Boni de Bobo-Dioulasso (UNB) du Burkina Faso, 01 BP 1091 Bobo-Dioulasso 01, \\ ${ }^{3}$ Laboratoire d'Etude et de Recherche des Ressources Naturelles et des Sciences de l'Environnement \\ (LERNSE/UNB \\ Adresse pour la correspondance : SANA Youssoufou, Courriel : ysana2@yahoo.fr cel : (+226) 70722787) \\ Original submitted in on $13^{\text {th }}$ February 2020. Published online at www.m.elewa.org/journals/ on $31^{\text {st }}$ May 2020 \\ https://doi.org/10.35759/JABs.149.11
}

\section{RESUME}

Objectif: Cette étude a pour but d'évaluer l'effet d'une alimentation à base de Brachiaria ruzienzis (Congo grass), Aeschynomene histrix (Joint-vetch porc épic), Stylosanthès hamata (Pencilflower), et Arachis pintoï (Arachide fourragère) sur les performances zootechniques des lapins.

Méthodologie et résultats : Le dispositif expérimental a été réalisé sur la Station de Recherche de FarakoBâ. Au total, neuf (9) lapins mâles (Oryctolagus cuniculus L.) âgés de dix (10) semaines et pesant en moyenne $(766,50 \pm 89,04 \mathrm{~g})$ chacun, ont été répartis dans 3 cages de volume : $42 \mathrm{~cm} \times 63 \mathrm{~cm} \times 39 \mathrm{~cm}$ soit un volume de $103194 \mathrm{~cm} 3$ chacune. Ces lapins ont été répartis en trois (3) lots de trois (3) lapins chacun. Les lots ont reçu les rations suivantes: la ration1du lot1(R1L1) était composée de l'aliment Brachiaria ruzienzis et Aeschynomene histrix; la ration 2 (R2L2), Brachiaria ruzienzis et Stylosanthès hamata et la ration 3 (R3L3), Brachiaria ruzienzis et Arachis pintoï. A chaque ration on a ajouté du son de maïs et de riz et du sel. Les lapins nourris à la ration R3L3, ont eu un gain moyen journalier de $5,72 \pm 2,74 \mathrm{~g} / \mathrm{j} / \mathrm{j}$ contre $6,18 \pm 3,04 \mathrm{~g} / \mathrm{j}$ pour R1L1 et $6,59 \pm 3,93 \mathrm{~g} / \mathrm{j}$ pour R2L2.

Conclusion et application des résultats : Les meilleurs variations des poids vifs finaux ont été obtenus chez les animaux nourris avec les rations R1L1 et R2L2 respectivement $223,83 \pm 45,67 \mathrm{~g}$ et $248,83 \pm 43,34 \mathrm{~g}$. Ces deux combinaisons ont également données les meilleurs GMQ. Sur la base du GMQ ces deux rations ont ainsi été plus efficientes que la ration R3L3. II n'y a pas eu de différence entre les trois rations $(p>0,5)$ mais la ration R2L2 donne de bonnes performances zootechniques et constitue un atout économique pour les cunicultures.

Mots clés : lapins, rations, gain moyen quotidien alimentation 


\section{ABSTRACT \\ Feeding of rabbits (Oryctolagus cuniculus L.) based on Brachiaria ruzienzis and Aeschynomene histrix, Stylosanthes hamata and, Arachis pintoï on zootechnical performance.}

Objective: This study aims to evaluate the effect of a diet based on Brachiaria ruzienzis(Congo grass), Aeschynomene histrix(Joint-vetch porc épic), Stylosanthès hamate (Pencilflower), and Arachis pintoï (Arachide fourragère) on the zootechnical performance of rabbits.

Methodology and results:The experimental device was carried out at the Farako-Bâ Research Station. Nine (9) male rabbits (Oryctolagus cuniculus L.) aged ten (10) weeks and weighing on average (766.50 $\pm 89.04 \mathrm{~g}$ ) each, were distributed in three volume cages: $42 \mathrm{~cm}$ x $63 \mathrm{~cm}$ x $39 \mathrm{~cm}$, each having a volume of $103194 \mathrm{~cm} 3$. These rabbits were divided into three (3) lots of three (3) rabbits each. The lots received the following rations: ration1 of Lot 1(R1L1) consisted of the food Brachiaria ruzienzis and Aeschynomene histrix; ration 2 (R2L2), Brachiaria ruzienzis and Stylosanthès hamata and ration 3 (R3L3), Brachiaria ruzienzis and Arachis pintoï. Corn and rice bran and salt was added to each ration. Rabbits fed R3L3, had an average daily gain of $5.72 \pm$ $2.74 \mathrm{~g} / \mathrm{j}$ versus $6.18 \pm 3.04 \mathrm{~g} / \mathrm{j}$ for R1L1 and $6.59 \pm 3.93 \mathrm{~g} / \mathrm{j}$ for R2L2.

Conclusion and application of results: The best variations in final live weights were obtained in animals fed with the R1L1 and R2L2 rations respectively $223.83 \pm 45.67 \mathrm{~g}$ and $248.83 \pm 43.34 \mathrm{~g}$. These two combinations also gave the best GMQs. Based on the $G M Q$ these two rations were thus more efficient than the R3L3 ration. There was no difference between the three rations $(p>0.5)$ but the R2L2 ration provides good zootechnical performance and is an economic asset for cuniculture.

Key words: rabbits, rations, average daily gain, feed

\section{INTRODUCTION}

Le Burkina Faso est un pays sahélien dont l'économie est essentiellement basée sur l'agriculture et l'élevage. Le secteur de l'élevage constitue une des bases du développement socioéconomique du pays. II contribue à environ $12 \%$ au produit intérieur brut (PIB) et représente près de $27,2 \%$ de la valeur ajoutée du pays. (MRA, 2007). Les productions animales sont en dessous des besoins d'une population en pleine expansion démographique (MAEP, 2008). L'élevage des lapins est un élevage qui génère beaucoup de revenu en peu de temps (Lebas, 2008). Sa viande a des qualités exceptionnelles qui la distinguent des autres. Sa chair est savoureuse et ressemble à celle du poulet. Elle est pauvre en graisses et en sodium et possède un bon indice d'instauration (Buldgen, 1996). Toutefois, la réussite d'un programme de développement cunicole suppose une amélioration

\section{MATERIELS ET METHODES}

Site d'étude : Le dispositif expérimental a été réalisé sur la Station de Recherche de Farako-bâ. Au total, neuf (9) lapins mâles (Oryctolagus cuniculus L.) âgés de 10 semaines de poids moyen $(766,50 \pm 89,04 \mathrm{~g})$ sont repartis dans 3 cages de volume: $126 \times 63 \times 39 \mathrm{~cm}^{3}$ de la santé et l'alimentation des lapins qui représentent 60 à $70 \%$ des coûts de production (Diallo et al., 1994). De nombreuses études ayant pour objectif principale l'amélioration de l'alimentation des lapins par l'usage ses ressources locales accessibles et disponibles ont été entreprises un peu partout en Afrique. Dans une étude récente (Mutwedu et al., 2015) ont montré que les éleveurs congolais font appel à plusieurs produits et sous-produits locaux pour alimenter leurs lapins. Les fourrages verts retrouvés un peu partout dans le pays sont les plus retrouvés dans la ration des lapins. L'objectif de ce travail est (i) d'étudier les performances zootechniques induites par des aliments formulés à base de Brachiaria ruzienzis et trois légumineuses, Aecheminès hitrix, Stylosanthès hamata, Arachida pinti et (ii) d'évaluer les performances zootechniques des lapins.

(longueur, largeur et hauteur). Chaque cage a trois compartiments mesurant $42 \times 63 \times 39 \mathrm{~cm}^{3}$ (longueur, largeur et hauteur). Les cages sont disposées au hasard dans deux bâtiments éclairés par la lumière. Chaque 
cage est munie d'une mangeoire en boîte de tomate et d'un abreuvoir en bidon.

Animaux: Le matériel animal utilisé se compose de neuf lapins de race locale, sevrés, âgés de dix (10) semaines et ayant un poids moyen $766,50 \pm 89,04 \mathrm{~g}$. Ces animaux provenant de l'unité de recherche cunicole de Farakobâ, ont été déparasités (interne et externe) avant l'expérience. Ils ont été identifiés individuellement par des numéros de cage et ont été répartis en 03 lots de 03 lapins chacun. Les batteries de cages étaient installées dans le bâtiment muni de claustras d'aération et d'un éclairage naturel et électrique. L'essai s'est déroulé sur deux périodes, une période d'adaptation de 7 jours et une période de collecte hebdomadaire de données durant 12 semaines.

Aliments : Trois aliments à base de Brachiaria ruzienzis constitués de Aeschynomene histrix, Stylosanthès hamata, et Arachis pintoï récoltés sur les parcelles de la station de Farakobâ et des compléments alimentaires constitués de son de riz et de maïs. Les rations (R1L1, R2L2, R3L3) étaient composées de $300 \mathrm{~g}$ de graminée de base fraiche, $150 \mathrm{~g}$ de légumineuse fraiche chacun, $25 \mathrm{~g}$ de son de riz, $25 \mathrm{~g}$ de son maïs et $10 \mathrm{~g}$ du sel.

Dispositif expérimental : Les rations expérimentales ont été distribuées chaque jour à $8 \mathrm{~h} 30 \mathrm{mn}$. Tous les matins, les quantités non consommées sont retirées des mangeoires et pesées pour chaque animal, le reste d'eau est vidé et remplacer par de l'eau propre. On a une phase d'adaptation d'une semaine suivi d'une phase de collecte de données pendant 12 semaines. La composition des rations étudiées (R1L1, R2L2, R3L3) est présentée dans le Tableau 1.

Tableau 1. Proportion alimentaire des rations(\%)

\begin{tabular}{lccc}
\hline Ingrédients & R1L1 & R2L2 & R3L3 \\
\hline Brachiaria ruzienzis & 58,82 & 58,82 & 58,82 \\
Aeschynomene histrix & 29,41 & & \\
Stylosanthès hamata & & 29,41 & 29,41 \\
Arachis pintoï & & & 4,90 \\
Son de maîs & 4,90 & 4,90 & 4,90 \\
Son de riz & 4,90 & 4,90 & 1,96 \\
22Sel & 1,96 & 1,96 & 100 \\
\hline Composition & 100 & 100 & \\
\hline
\end{tabular}

Suivi de la consommation alimentaire et pondérale :Une période d'adaptation de 7 jours a précédé le début de mesure des performances pour permettre aux animaux de s'habituer aux rations expérimentales

\section{$\checkmark \quad$ La consommation alimentaire}

La consommation alimentaire ou quantité d'aliment ingéré (QAI) a été calculée à partir des quantités d'aliments distribuées et les quantités refusées. Les aliments offerts ont été pesés avant d'être distribués le matin et les refus de chaque animal ont été collectés et pesés tous les matins avant la distribution de la ration du jour.

$\checkmark \quad$ Le poids vif (en $\mathrm{kg}$ )

Le poids vif (PV) a été mesuré par des pesées chaque semaine à l'aide d'un peson de $5 \mathrm{~kg}$. Les pesées ont été faites à jeun le matin avant la distribution de la ration du jour.

Evaluation du gain moyen quotidien (GMQ) et de l'indice de consommation (IC): Le Gain Moyen Quotidien (GMQ) indique la vitesse moyenne de croissance pendant une période déterminée. II a été calculé selon l'Équation 1. Quant à l'Indice de consommation (IC) qui est un nombre sans unité, il traduit l'efficacité de l'utilisation alimentaire sur la période étudiée. Autrement, c'est la quantité d'aliment consommée par l'animal pendant une période donnée pour produire $1 \mathrm{Kg}$ de chair. L'IC est calculé selon l'Équation 2.

$$
\begin{aligned}
& \text { GMQ }=\frac{\text { Poids Final }(\mathrm{PF})-\text { Poids Initial }(\mathrm{PI})}{\begin{array}{l}
\text { Nombre de jour } \\
(1)
\end{array}} \\
& \mathrm{IC}=\frac{\mathrm{QAI}(\mathrm{g}) \text { sur une période considérée }}{\text { Gain de poids }(\mathrm{g}) \text { sur la même période }}
\end{aligned}
$$

Détermination de la composition chimique : Elles ont été effectuées au Laboratoire d'analyse GRN de la station de Farakobâ et au Laboratoire de Nutrition Animale au Centre de Recherches Environnementales Agricoles et de Formation (CREAF) de I'INERA à Kamboinsé. Elles ont concerné les échantillons des 
coques de niébé et aliments distribués. Sur les différents échantillons, nous avons déterminé:

$\checkmark \quad$ la Matière Sèche (MS) obtenue par séchage à $105^{\circ} \mathrm{C}$ dans une étuve pendant 24 heures ;

$\checkmark \quad$ la Matière Minérale (MM) ou cendres par passage de l'échantillon sec dans un four à $550^{\circ} \mathrm{C}$ pendant 3 heures ;

$\checkmark \quad$ la Matière Organique (MO) obtenue par différence entre la MS et les cendres (MM);

$\checkmark \quad$ la Matière Azotée Totale (MAT) par la méthode classique de KJELDAHL. Selon cette méthode, une minéralisation suivie d'une distillation permet obtenir le pourcentage d'azote de l'échantillon. La MAT est ensuite estimée en appliquant au pourcentage d'azote (\% N), le coefficient 6,25 conventionnellement utilisé ;

\section{RESULTATS}

Composition chimique des aliments: L'analyse bromatologique montre que la composition chimique varie selon le type d'aliment.La teneur en matière sèche des aliments utilisés au cours de l'essai est comprise entre 92,50 et $93,96 \%$. Les rations issues des sous-
Analyses statistiques : Les données collectées ont été saisies sur le tableur Excel version 2010. L'analyse de ces données a été effectuée à l'aide du logiciel $R{ }^{2}$. L'analyse des variances (ANOVA) a été appliquée. Le test de Bartlett ou celui de Student Newman et Keuls au seuil de $5 \%$ ont été utilisés pour la séparation des variances lorsque l'analyse relevait une différence entre les moyennes. Par ailleurs, lorsque cela a été nécessaire, la méthode de Bonferronni a été utilisée pour la correction des probabilités comme recommandé en cas de tests répétés (Rice, 1989). Les graphiques et les tableaux ont été tracés à l'aide du tableur Excel version 2010.

produits de maïs et de riz renferment $6,79 \%$ à $16 \%$ de matière azotée totale. Le taux de MAT 16\% de Arachis pintoï est supérieur aux deux autres légumineux Aecheminès hitrix et Stylosanthès hamata, respectivement $14 \%$ et $12 \%$ (Tableau 2).

Tableau 2: Composition chimique des aliments

\begin{tabular}{|l|l|l|l|}
\hline Aliments & MS\% & MAT (\%MS) & MM\% \\
\hline Brachiaria ruzienzis & 92,50 & 8,50 & 9,40 \\
\hline Aecheminès hitrix & - & 14,00 & 5,15 \\
\hline Stylosanthès hamata & - & 12,00 & 10,00 \\
\hline Arachis pintoï & - & 16,00 & 10,00 \\
\hline Son de maîs & 93,96 & 6,79 & 4,04 \\
\hline Son de riz & 93,51 & 7,01 & 6,76 \\
\hline
\end{tabular}

Quantité de matière sèche consommée : Les valeurs de la consommation alimentaire bihebdomadaire moyenne minimum des rations obtenues au début de l'expérience sont $174,90 \pm 17,82 \mathrm{~g} ; 77,1 \pm 7,64 \mathrm{~g}$ et $157,20 \pm 58,97 \mathrm{~g}$ respectivement pour la ration $\mathrm{R} 1 \mathrm{~L} 1$, la ration $\mathrm{R} 3 \mathrm{~L} 3$, et la ration $\mathrm{R} 2 \mathrm{~L} 2$ (Tableau 3 ). Entre le 10ème et la $12^{\mathrm{ème}}$ semaine, les valeurs moyennes maximales de la consommation alimentaire obtenues sont $201,90 \pm$ $14,42 \mathrm{~g}, \quad 232,20 \pm 10,61 \mathrm{~g}$ et $118,80 \pm 2,97 \mathrm{~g}$ respectivement pour la ration $\mathrm{R} 1 \mathrm{~L} 1$, la ration $\mathrm{R} 2 \mathrm{~L} 2$ et la ration $\mathrm{R} 3 \mathrm{~L} 3$. La différence est non significative entre la consommation alimentaire moyenne bihebdomadaire des rations R1L1 et R2L2 ( $p>0,05)$. Par contre une différence significative a été observée entre ces deux rations et la ration $R 3 L 3$. Le coefficient de variation de la consommation de la ration R1L1 est supérieur $(0,068)$ aux deux rations $R 2 L 2, R 3 L 3$ respectivement $(0,181)$ et $(0,222)$. La Figures 1 montre respectivement l'évolution (a) et la variabilité (b) de la consommation moyenne des trois rations (R1L1, R2L2, R3L3). 
Tableau 3: Évolution de la consommation alimentaire bihebdomadaire des rations

\begin{tabular}{lllll}
\hline \multirow{4}{*}{ Semaines } & \multicolumn{4}{l}{ Consommation moyenne alimentaire bihebdomadaire } \\
\cline { 2 - 5 } & R1L1 & R2L2 & R3L3 & Pr (>F) \\
\hline S2 & $174,90 \pm 17,82$ & $157,20 \pm 58,97$ & $77,10 \pm 7,64$ & $0,0622$. \\
S4 & $191,70 \pm 5,94$ & $145,20 \pm 40,31$ & $64,50 \pm 16,12$ & $0,0471^{*}$ \\
S6 & $210,90 \pm 5,94$ & $207,90 \pm 16,12$ & $88,50 \pm 26,30$ & 0,9754 \\
S8 & $185,10 \pm 37,34$ & $219,60 \pm 2,97$ & $77,40 \pm 22,49$ & 0,5861 \\
S10 & $201,90 \pm 14,42$ & $205,80 \pm 11,46$ & $101,10 \pm 7,64$ & 0,55861 \\
S12 & $183,90 \pm 7,64$ & $232,20 \pm 10,61$ & $118,80 \pm 2,97$ & 0,5945 \\
Moyenne & $191,40 \pm 13,10 \mathrm{a}$ & $194,65 \pm 35,16 \mathrm{a}$ & $87,90 \pm 19,51 \mathrm{~b}$ & $1,559 \mathrm{e}-066^{* * *}$ \\
CV & 0,068 & 0,181 & 0,222 & \\
\hline
\end{tabular}

La ration R1L1 contient le Brachiaria ruzienzis et Aecheminès hitrix, la ration R2L2 contient Brachiaria ruzienzis et Stylosanthès hamata, la ration R3L3 contient Brachiaria ruzienzis et Arachis pintoï. Sur la même ligne, les lettres abc indique l'appartenance à des groupes différents suivant le test de Student Newman et Keuls au seuil de $5 \%$. Les codes de significativité ont été les suivants . $\mathrm{P}<0,1 ;{ }^{*}: \mathrm{P}<0,05 ;{ }^{* *}: \mathrm{P}<0,01 ;{ }^{* *}: \mathrm{P}<0,001$

La variation de la consommation des lapins en fonction des rations a été déterminée. II ressort que la consommation des lapins évolue en dent de scie de la première à la $12^{\mathrm{ème}}$ semaine pour les rations.

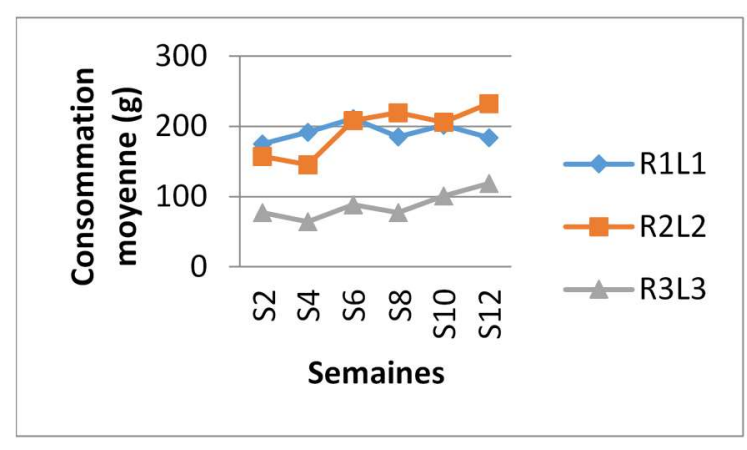

(a)

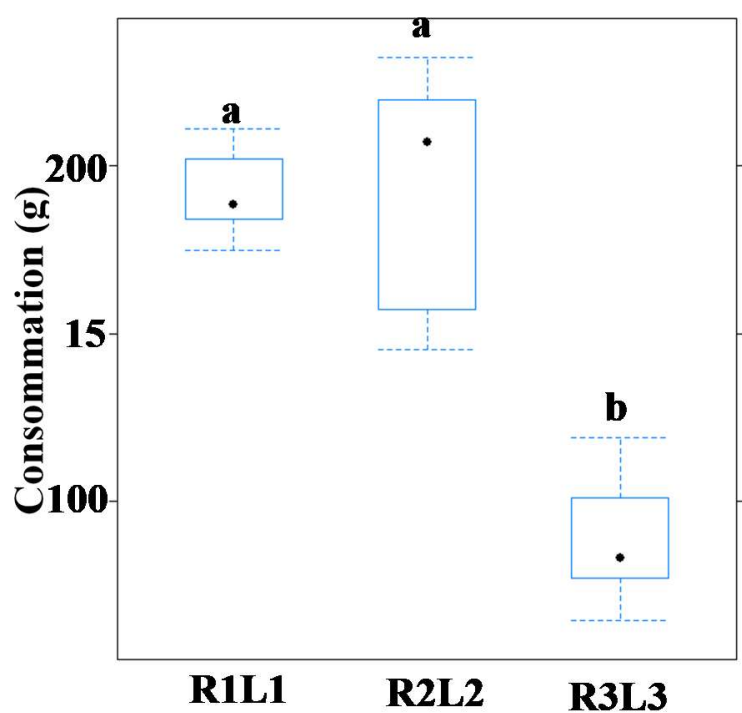

(b)

Figure 1 : Consommation bihebdomadaire (a) évolution et (b) variabilité

Évolution du poids vif corporel des lapins : En général, la croissance pondérale des lapins a été progressive du début à la fin de l'expérimentation pour la ration $\mathrm{R} 1 \mathrm{~L} 1$. De même, le poids le plus élevé a été obtenu à la 12ème semaine avec les rations R1L1 et R2L2 (Tableau 4).Nous notons cependant, une diminution du poids vif au niveau de la $10^{\text {ème }}$ semaine pour la ration R3L3. A la quatrième semaine on observe une différence significative entre les rations $(p<0,05)$. A partir de la sixième une différence non significative a été observé
.Les mêmes tendances ont été observés jusqu'à la fin de l'expérience. Les poids vifs corporels moyen ne montrent pas une différence significative entre les deux rations (R2L2 et R1L1). Ces deux ont une différence significative avec R3L3 $(p<0,05)$. Le coefficient de variation de la ration R3L3 0,215 est supérieur aux deux rations $R 2 L 2$ et $R 1 L 1$ respectivement 0,204 et 0,174. La Figure 2 montre respectivement l'évolution (a) et la variabilité (b) de poids vif corporel moyen des trois rations (R1L1, R2L2, R3L3). 
Tableau 4: Évolution des poids vif corporel (g) bihebdomadaires des lapereaux.

\begin{tabular}{lllll}
\hline Semaines & \multicolumn{2}{l}{ Poids vif corporel bihebdomadaire } & \multirow{2}{*}{$\operatorname{Pr}(>\mathrm{F})$} \\
\cline { 2 - 4 } & $\mathbf{R}$ R1 & R2L2 & R3L3 & \\
\hline S2 & $168,12 \pm 25,87$ & $198,87 \pm 56,21$ & $70,28 \pm 12,28$ & $0,00363^{* *}$ \\
S4 & $198,23 \pm 56,23$ & $204,65 \pm 25,78$ & $90,36 \pm 24,13$ & $0,02310^{*}$ \\
S6 & $201,54 \pm 28,47$ & $230,28 \pm 54,28$ & $107,87 \pm 35,27$ & 0,10542 \\
S8 & $230,45 \pm 65,58$ & $280,87 \pm 25,54$ & $121,78 \pm 47,21$ & 0,96956 \\
S10 & $248,57 \pm 55,87$ & $291,24 \pm 41,32$ & $90,65 \pm 25,25$ & 0,96956 \\
S12 & $298,35 \pm 98,58$ & $290,69 \pm 45,28$ & $128,87 \pm 27,25$ & 0,11963 \\
Moyenne & $223,83 \pm 45,67 a$ & $248,83 \pm 43,34 a$ & $101,00 \pm 21,76 \mathrm{~b}$ & $1,529 \mathrm{e}-05^{* * *}$ \\
CV & 0,204 & 0,174 & 0,215 & \\
\hline
\end{tabular}

La ration R1L1 contient le Brachiaria ruzienzis et Aecheminès hitrix, la ration R2L2 contient Brachiaria ruzienzis et Stylosanthès hamata, la ration R3L3 contient Brachiaria ruzienzis et Arachis pintoï. Sur la même ligne, les lettres abc indique l'appartenance à des groupes différents suivant le test de Student Newman et Keuls au seuil de 5\%. Les codes de significativité ont été les suivants . $\mathrm{P}<0,1 ;{ }^{*}: \mathrm{P}<0,05 ;{ }^{* *}: \mathrm{P}<0,01 ;{ }^{* * *}: \mathrm{P}<0,001$

La variabilité des poids vifs corporels des lapins en fonction des rations a été déterminée. II ressort que le poids des lapins a augmenté progressivement de la première à la $8^{\text {ème }}$ semaine avec un chut à la 10 ème pour la ration $\mathrm{R} 3 \mathrm{~L} 3$.

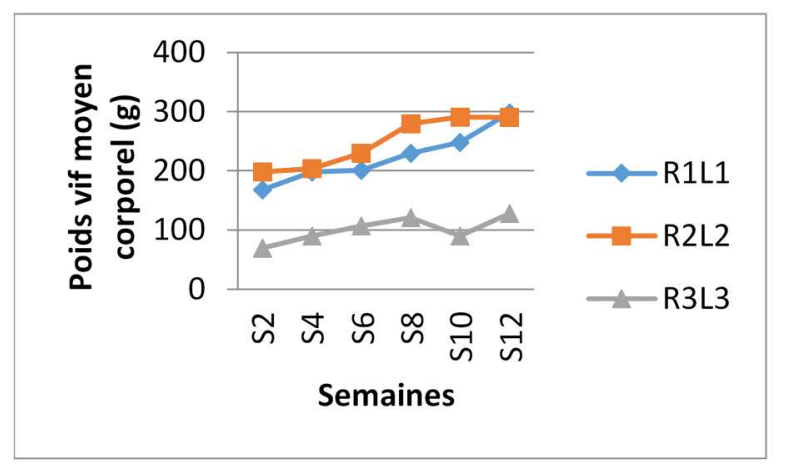

(a)

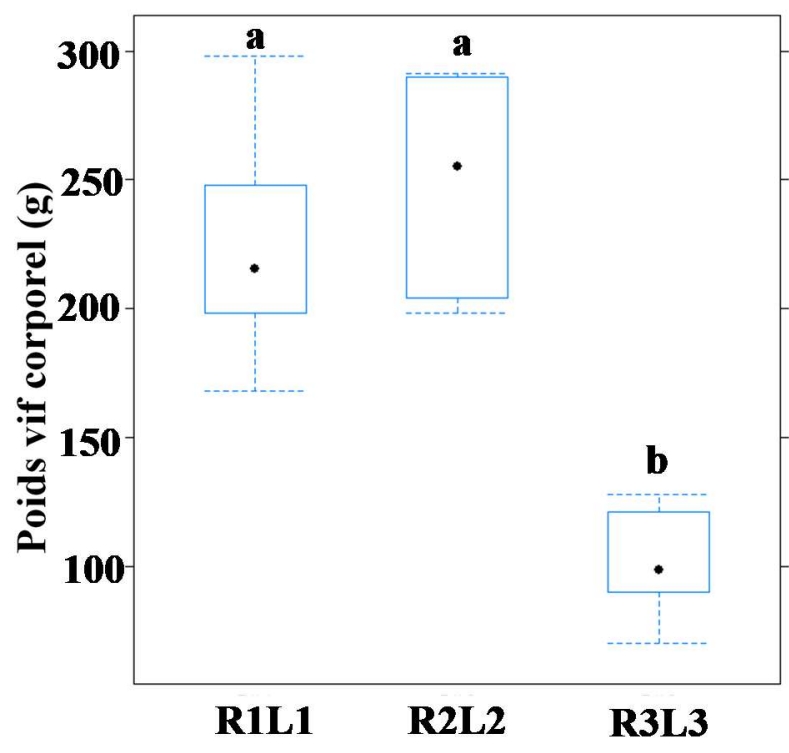

(b)

Figure 2 : Poids vif corporel(g) bihebdomadaires (a) évolution et (b) variabilité

Performance des lapins durant l'essai : Au début de l'expérimentation une différence significative $(p<0,01)$ a été observée entre les poids vifs moyens au 14ème jour $(168,12 \pm 25,87 \mathrm{~g}, 198,87 \pm 56,21 \mathrm{~g}$ et $70,28 \pm 12,28 \mathrm{~g})$ des animaux nourris avec les trois rations alimentaires (R1L1, R2L2, R3L3) respectivement. A la fin de l'expérience il n'avait pas de différence significative entre les poids finaux des lapins nourris avec les rations (R1L1 et R2L2) respectivement 223,83 $\pm 45,67,248,83 \pm$ $43,34 \mathrm{~g}(\mathrm{p}>0,05)$. La différence a été non significative entre les GMQ moyens des animaux nourris avec les trois rations R1L1, R2L2 et R3L3 au seuil de 5\% (Tableau 5). Les résultats du gain moyen quotidien montrent une différence significative à 14 jours 
d'expérience $(p>0,001)$. Le coefficient de variation de la ration R2L2 0,596 est supérieur aux deux rationsR1L1 et R3L3. La Figures 3 montre respectivement l'évolution (a) et la variabilité (b) des GMQ moyens des trois rations (R1L1, R2L2, R3L3).

Tableau 5: Évolution des GMQ (g/j) bihebdomadaires des lapins durant l'essai

\begin{tabular}{lllll}
\hline \multirow{4}{*}{ Semaines } & \multicolumn{3}{l}{ GMQ bihebdomadaires } & \\
\cline { 2 - 3 } & R1L1 & R2L2 & R3L3 & \\
S2 & & & & \\
S4 & $12,00 \pm 5,25$ & $14,14 \pm 4,29$ & $10,71 \pm 4,28$ & $6,35 \mathrm{e}-08^{* * *}$ \\
S6 & $7,07 \pm 2,54$ & $7,29 \pm 3,57$ & $6,96 \pm 2,24$ & $0,000393^{* * *}$ \\
S8 & $4,79 \pm 2,54$ & $5,48 \pm 2,36$ & $4,93 \pm 1,27$ & 0,118174 \\
S10 & $4,46 \pm 2,56$ & $5,00 \pm 2,14$ & $4,48 \pm 1,58$ & 0,341688 \\
S12 & $4,26 \pm 2,58$ & $4,16 \pm 1,87$ & $3,79 \pm 1,09$ & 0,341688 \\
Moyenne & $4,49 \pm 2,58$ & $3,45 \pm 1,25$ & $3,46 \pm 1,87$ & 0,659130 \\
CV & $6,18 \pm 3,04 a$ & $6,59 \pm 3,93 a$ & $5,72 \pm 2.74 a$ & 0,9016 \\
\hline
\end{tabular}

La ration R1L1 contient le Brachiaria ruzienzis et Aecheminès hitrix, la ration R2L2 contient Brachiaria ruzienzis et Stylosanthès hamata, la ration R3L3 contient Brachiaria ruzienzis et Arachis pintoï. Sur la même ligne, les lettres abc indique l'appartenance à des groupes différents suivant le test de Student Newman et Keuls au seuil de $5 \%$. Les codes de significativité ont été les suivants $\cdot P<0,1 ;{ }^{*}: P<0,05 ;{ }^{* *}: P<0,01 ;{ }^{* *}: P<0,001$

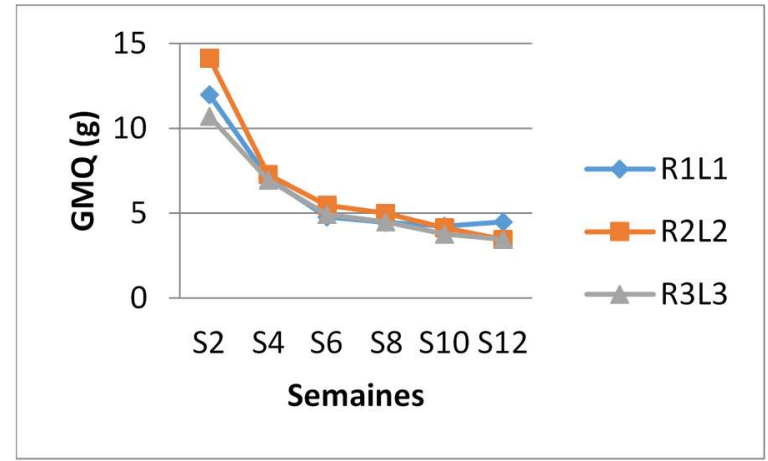

(a)

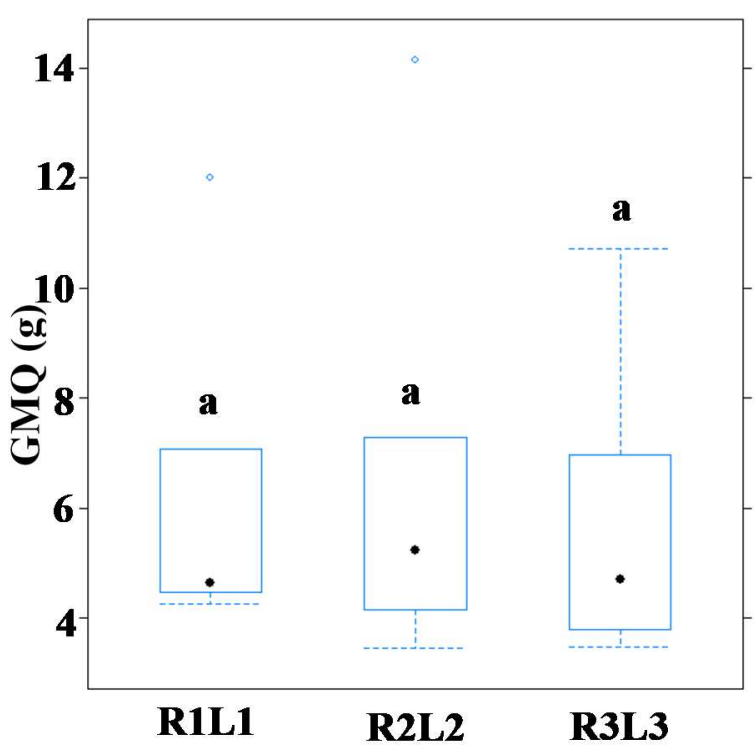

(b)

Figure 3: GMQ bihebdomadaires (a) évolution et (b) variabilité

La différence entre la consommation alimentaire bihebdomadaire moyenne des animaux nourris avec les rations alimentaires R1L1, R2L2 et R3L3 respectivement $191,40 \pm 13,10 \mathrm{~g} ; 194,65 \pm 35,16 \mathrm{~g}$ et $87,90 \pm 19,51 \mathrm{~g}$ (Tableau 6). Une différence non significative a été observée entre les rations R1L1 et R2L2. Par contre la ration R3L3 la a été significative différente par rapport aux deux rations R1L1 et R2L2 $(P>0,05)$. Les indices de consommation moyens sont $0,88 \pm 0,17 ; 0,78 \pm 0,07$ et $0,89 \pm 0,20$ respectivement pour les rations R1L1, R2L2 et R3L3. On observe une différence non significative entre les trois rations $(P>0,05)$. Le coefficient de variation de la ration R3L3 $(0,223)$ est supérieur aux des rations R1L1et R2L2 
respectivement $(0,191$ et 0,092). La Figures 4 montre respectivement l'évolution (a) et la variabilité (b) des indices de consommation moyenne des trois rations (R1L1, R2L2 et R3L3).

Tableau 6 : Évolution des indices de consommations des lapins durant l'essai

\begin{tabular}{lllll} 
Semaines & \multicolumn{2}{l}{ Indice de consommation } & Pr (>F) \\
\cline { 2 - 4 } & R1L1 & R2L2 & R3L3 & \\
\hline S2 & $1,04 \pm 0,17$ & $0,79 \pm 0,09$ & $1,10 \pm 0,03$ & 0,458 \\
S4 & $0,97 \pm 0,16$ & $0,71 \pm 0,07$ & $0,72 \pm 0,07$ & 0,526 \\
S6 & $1,05 \pm 0,12$ & $0,90 \pm 0,03$ & $0,83 \pm 0,06$ & 0,727 \\
S8 & $0,80 \pm 0,09$ & $0,78 \pm 0,06$ & $0,64 \pm 0,04$ & 0,297 \\
S10 & $0,81 \pm 0,05$ & $0,71 \pm 0,07$ & $1,12 \pm 0,07$ & 0,280 \\
S12 & $0,62 \pm 0,14$ & $0,80 \pm 0,09$ & $0,93 \pm 0,06$ & 0,448 \\
Moyenne & $0,88 \pm 0,17 a$ & $0,78 \pm 0,07 a$ & $0,89 \pm 0,20 a$ & 0,4416 \\
CV & 0,191 & 0,092 & 0,223 & \\
\hline
\end{tabular}

La ration R1L1 contient le Brachiaria ruzienzis et Aecheminès hitrix, la ration R2L2 contient Brachiaria ruzienzis et Stylosanthès hamata, la ration R3L3 contient Brachiaria ruzienzis et Arachis pintoï. Sur la même ligne, les lettres abc indique l'appartenance à des groupes différents suivant le test de Student Newman et Keuls au seuil de 5\%. Les codes de significativité ont été les suivants : . $P<0,1 ;{ }^{*}: P<0,05 ;{ }^{* *}: P<0,01 ;{ }^{* * *}: P<0,001$

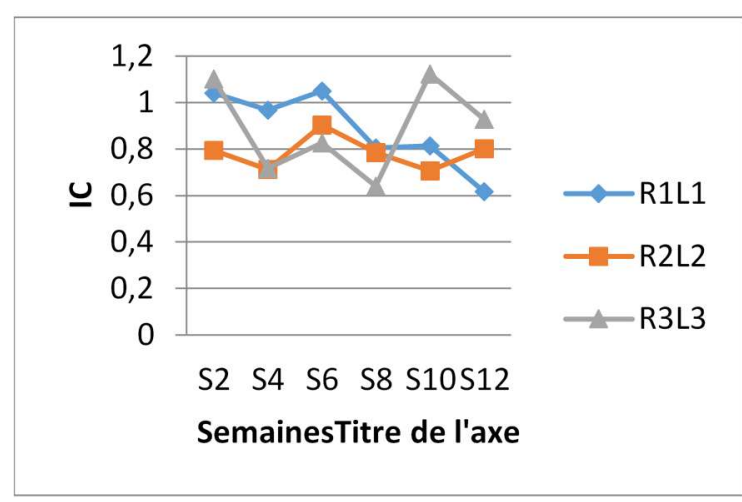

(a)

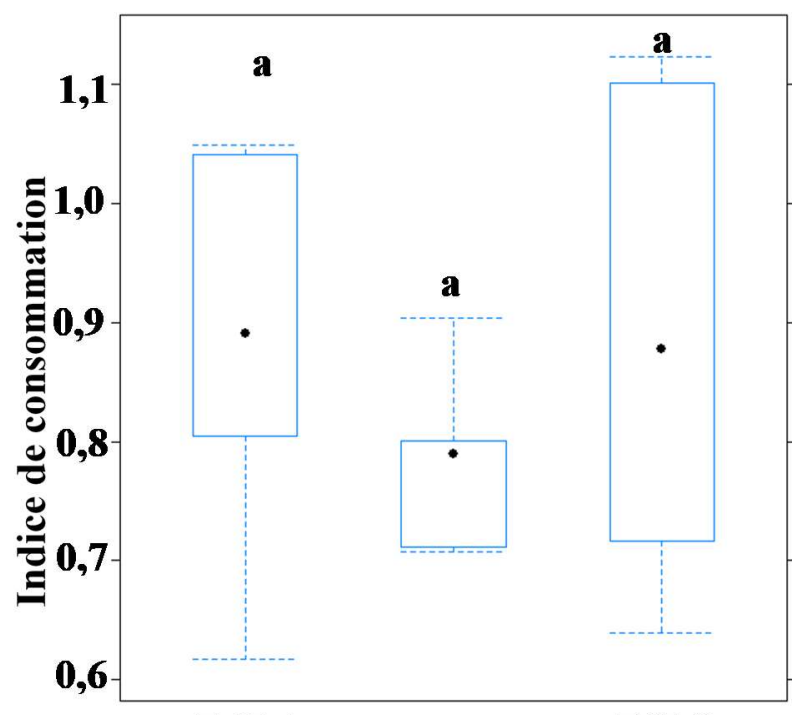

R1L1 R2L2 R3L3

(b)

Figure 4 : Indice de consommation bihebdomadaire (a) évolution et (b) variabilité

Une synthèse des résultats de performances ont été consignés dans le Tableau 7. Les poids moyens finaux sont $948,35 \pm 25,50 \mathrm{~g}, 1160,69 \pm 41,2 \mathrm{~g}, 958,87 \pm 35,25 \mathrm{~g}$ respectivement pour les rations $\mathrm{R} 1 \mathrm{~L} 1, \mathrm{R} 2 \mathrm{~L} 2$ et $\mathrm{R} 3 \mathrm{~L} 3$.

Tableau7 : Performances des lapereaux nourris avec les différentes rations alimentaires 


\begin{tabular}{llll}
\hline Paramètres & R1L1 & R2L2 & R3L3 \\
\hline Poids moyen initial(g) & $650,00 \pm 40,88$ & $870,00 \pm 20,30$ & $830,00 \pm 0,00$ \\
Poids moyen final(g) & $948,35 \pm 25,50$ & $1160,69 \pm 41,23$ & $958,87 \pm 35,25$ \\
moyenne de poids $(\mathrm{g})$ & $223,83 \pm 45,67 \mathrm{a}$ & $248,83 \pm 43,34 \mathrm{a}$ & $101,00 \pm 21,76 \mathrm{~b}$ \\
Gain moyen quotidien (g/j) & $6,18 \pm 3,04 \mathrm{a}$ & $6,59 \pm 3,93 \mathrm{a}$ & $5,72 \pm 2,74 \mathrm{a}$ \\
Consommation alimentaire quotidienne $(\mathrm{g})$ & $191,40 \pm 13,10 \mathrm{a}$ & $194,65 \pm 35,16 \mathrm{a}$ & $87,90 \pm 19,51 \mathrm{~b}$ \\
Indice de consommation & & & \\
& $0,88 \pm 0,17 \mathrm{a}$ & $0,78 \pm 0,07 \mathrm{a}$ & $0,89 \pm 0,20 \mathrm{a}$ \\
\hline
\end{tabular}

\section{DISCUSSION}

Valeurs nutritives des rations: Les compositions chimiques des rations alimentaires expérimentales sont comparables aux valeurs trouvées dans la littérature. Les taux de protéine brute obtenus dans cette étude pour les rations alimentaires expérimentales se situent dans l'intervalle 10,45 à $21,90 \%$ et sont similaires à ceux trouvés par Wogar et Ayuk (2012) et par Wogar et al. (2013). De même, les teneurs de cendre brute obtenues pour les trois rations alimentaires expérimentales sont comprises entre 9,40 et 10,00\%. Ces teneurs sont conformes à celles recommandées par Mensah, 1993 et Mensah, 1995. Les teneurs en matières sèches (92,50$93,96 \% \mathrm{MS})$, en cendres brutes $(9,40-10,00 \mathrm{MS})$ obtenues dans cette étude sont similaires à celles rapportées par Traoré et al. (2009). Les teneurs en cendres brutes obtenues (9,10-9,96\%) par Banjo et al. (2012) et $(6,72-9,31 \%)$ par Wogar et al. (2013) sont similaires à nos résultats.

Consommation alimentaire et croissance pondérale des lapins: Les valeurs de la consommation alimentaire bihebdomadaire moyenne des rations alimentaires R1L1, R2L2 et R3L3 obtenues sont similaires aux auteurs (Mensah, 1995), Uwalaka et Ahaotu (2013) comprises entre 150 et $250 \mathrm{~g}$. Elles sont également supérieures à celles obtenues par Ansah et al. (2012) qui étaient comprises entre 102,74 et 116,31 $\mathrm{g}$ et Pokou et al. (2013) qui étaient également comprises entre 114 et $115 \mathrm{~g}$. Les consommations journalières dans la première et deuxième semaine sont respectivement de $64,7 \pm 8,1 \mathrm{~g} / \mathrm{jr}$ et $76,60 \pm 12,20 \mathrm{~g} / \mathrm{jr}$ pour les lapins ayant reçu une alimentation contenant $8 \%$ de tourteau de coton. Djago et al. (2007), ont démontrés que la consommation d'un lapereau en engraissement est de 100 à $120 \mathrm{~g} / \mathrm{j}$. Dans notre étude, les consommations moyennes des trois rations (R1L1, R2L2 et R3L3) sont respectivement 191,40 $\pm 13,10 \mathrm{~g} / \mathrm{j}$,
$194,65 \pm 35,16 \mathrm{~g} / \mathrm{j}, 87,90 \pm 19,51 \mathrm{~g} / \mathrm{j}$. Nos résultats sont inférieurs à ceux trouvés sana et al, 2019 alimenté à base de Panicum maximum local. Cette différence pourrait s'expliquer par la différence entre les espèces (Panicum maximum local et Brachiaria ruzienzis) au niveau de la valeur nutritive et la morphologie. En effet, Lebas (2009) a montré que la consommation alimentaire dépend fortement de l'âge des lapins. L'accroissement du poids corporel obtenu peut être associé au taux élevé de protéine dans le régime. Selon Minson (1997), la teneur en protéine influence la quantité d'aliment consommé par les herbivores. Ceci est en accord avec nos résultats. En effet, les lapins ont plus consommé les rations alimentaires R1L1 et R2L2 par rapport à la ration alimentaire R3L3. La consommation alimentaire volontaire par l'animal peut baisser par rapport aux taux de protéines de la ration. Des observations similaires ont été faites chez les monogastriques herbivores (Annor et al., 2008 ; Poku et al., 2013). Minson (1997) ont rapporté que si le taux de protéine brute dans la ration est en dessous de 6-8\%, l'appétit de l'animal peut être diminué par la carence en protéine. Les taux de protéine contenu dans les trois rations alimentaires sont audessus de $8 \%$. Nous pouvons en déduire que les animaux ont eu l'appétit suffisant pour consommer les trois rations alimentaires.

Performance des lapins durant l'essai : L'analyse des résultats montre une différence non significative entre les poids moyens finaux des animaux nourris des deux rations alimentaires (R1L1 et $R 2 L 2$ ) par rapport aux poids moyen final des lapins nourris avec la ration alimentaire R3L3. Pour la consommation alimentaire bihebdomadaire, on n'a pas une différence significative entre les rations R1L1, R2L2. Par contre ces deux rations montrent une différence significative avec la ration R4L3. Les coefficients de variation de poids 
moyen obtenus des rations (R1L1, R3L3) sont supérieurs à la ration $\mathrm{R} 2 \mathrm{~L} 2$. Nos résultats sont similaires à (Amadou, 2014). L'indice de consommation alimentaire obtenus sont respectivement $0,88 \pm 0,17$; $0,78 \pm 0,07$ et $0,39 \pm 0,090,89 \pm 0,20$ respectivement pour les rations R1L1, R2L2 et R3L3. Ces indices sont inférieurs à ceux obtenus par Alida et Michèle (2013) qui étaient 5,3 $\pm 0,32$. Aussi, Akoutey et al. (2012) ont trouvé

\section{CONCLUSION ET APPLICATION DES RESULTATS}

L'utilisation des rations à base de Brachiaria ruzienzis produits localement dans l'alimentation des lapins a montré que les trois légumineux (Aeschynomene histrix, Stylosanthès hamata et, Arachis pintoï) influencent différemment le comportement alimentaire et les performances zootechniques des lapins. Les lapins peuvent être nourris uniquement avec les fourrages, les produits et sous-produits de maïs et du riz. Les meilleurs variations des poids vifs finaux ont été obtenus chez les animaux nourris avec les rations R1L1 et R2L2 respectivement $223,83 \pm 45,67 \mathrm{~g}$ et $248,83 \pm 43,34 \mathrm{~g}$. Ces

\section{REFERENCES BIBLIOGRAPHIQUES}

Akoutey A, Kpodekon H. 2012: Performances zootechniques de lapereaux recevant des aliments granulés contenant du Pueraria phaseoloides. Tropicultura, 30 : 161-166.

Alida MSY et Michel A, 2013: Effets de la supplémentation de Bocrhaviaerecia et de Portula caoleracea sur la croissance ponderale des lapereaux sevrés 40p.

Amadou M. 2014 : Etude in vivo de la digestibilité des coques de nébé(Vigna unguiculata) dans l'alimentation des lapins de race locale élévés en milieu tropical.Ecole polytechnique d'Abomey Calavi Diplôme de licence professionnelle en production et santé Animale 33p.

Annor AY, Kagya Agyeang JK, Abbam JEY, Oppong SK, Agoe IM: 2008 growth performance of grass cutter (Thryonomys swinderianus) eating leaf and stem fractions of Guinea grass (Panicum maximum) Levestock Research Rural Development, 20(8):125.

Banjo OS, Mako AA. and Ettu RO : 2012. The Replacement of Maize with graded level of brewer's Dried Grain (BDG) in diet of weaner grass cutters. Journal of Natural sciences research., 2 (8) : 186-190. un indice de consommation de $3,60 \pm 0,08$ et Amadou (2014) a obtenu des valeurs de $3,83 \pm 1,93 ; 2,51 \pm 0,83$; $2,68 \pm 1,15$ et $2,67 \pm 1,70$ sur les cosses de niébé à des proportions variables. Les résultats obtenus sana et al, 2019 à base de Panicum maximum local et les trois légumineux donnent des indices de consommations inférieures $0,64 \pm 0,17,0,50 \pm 0,12$ et $0,39 \pm 0,09$ respectivement $R 1 L 1, R 2 L 2$ et $R 3 L 3$.

deux combinaisons ont également données les meilleurs GMQ. Sur la base du GMQ ces deux rations ont ainsi été plus efficientes que la ration R3L3. Toutefois, les indices de consommation ont été relativement non significatifs entre les trois rations. Parmi les rations expérimentales, sur la base de l'indice de consommation, les résultats semblent montrer une meilleure valorisation de la ration R2L2. Ainsi, au regard de ces résultats et en guise de perspectives, il conviendrait de nourrir les lapins avec la ration R2L2 pour obtenir une meilleure croissance.

Buldgen 1996. Aviculture semi- industrielle en climat tropical : Guide pratique. Gembloux: les Presses agronomiques de Gembloux. $112 p$

Diallo K, Deravinia A, Bahus J. Elevage intensive : Perspective après la dévaluation : le défi de l'alimentation avicole. Afrique Agriculture, (212) 1994: 20-40.

Djago AY, Kpodekon, Lebas F. 2007. Associations "cunicultur" 31450 Corron sac- franc Document http://wwwcuniculture.info/Doc/Elevage/Tropi$01 \mathrm{htm}$

Lebas F., 2009 Maîtrise des conditions d'ambiance en élevage cunicole. Réunion formation GIPAC Tunisie 32p.

MAEP, 2008 Mécanisme Africain d'Evaluation par les Pairs : Rapport d'Evaluation du Burkina pays $n^{\circ} 9$ Mai, 2008, 276p.

Mensah GA 1993 Futterauf nahme und verdaulichkcit beim grasnager (Thryonomys winderianus) Thèse de doctorat institute 480 Université de Holenhein Allemagne 207p.

Mensah GA 1995 Consommation et digestibilité alimentaire chez les aulacode Thryonomys swinderiances. Tropicultura, 13. 123-124.

Minson DJ, 1997 Ruminants the protein producers Biologist, 44:463-464.

MRA/IDGPSE., 2007. Les statistiques du secteur de l'élevage au Burkina Faso, 70p. 
Mutwedu VB, Ayagirwe RBB, Metre KT, Mugumaarhahama Y, Sadiki JM, Bisimwa EB, 2015. Systèmes de production cunicole en milieu paysan au Sud-Kivu, Est de la RD Congo ; Livestock Research for Rural Development 27 (10) $2015 ; 14 p$

Poku JNPA, Annor SV, and Djang-Fordjour KT, 2013: Growth reproduction and carcass characteristics of grass cutters (Thryonomys Swinderianus) fed on different levels of protein supplement. World journal of zoology 8(2): 175184, 2013.

Uwalaka RE and Ahaotu EO, 2013: Performance of growing grass cutters fed on differend fibres Sources: international Journal of Veterinary Sciences 2(3):85-87 w.w.w.jijvets.com.

Sana Y. Sanou J., Kondombo S.R., Sawadogo L.et Kabore-Zoungrana C. Alimentation de trois types de rations alimentaires en vrac a base de fourrages verts et de son (maïs et riz) chez les lapins. Rev. Ivoir. Sci. Technol., 34 (2019) 405 - 420 ISSN 1813-3290, http://www.revist.ci

Traore B, Fantodji A, Mensah GA, 2009 Influence de la forme physique des aliments sur la croissance et le rendement en carcasse de Thryonomys swinderianus à trois stades physiologiques. Bulletin de la Recherche Agronomique du Benin N 65 Septembre 2009 1-31.

.Wogar GSI, Ufot ML, Henry AJ, Inyang IE et Efe EE. 2013 Composition and emulsifying Characteristics of grass cutters meat from varying Dietary levels. Journal of Agricultural.

Wogar GSI, Ayuk AA : 2012. By-Products as Protein Source for Lactating Grass cutters. Journal of Agricultural Science, 4(7): 148-153. 